\title{
A Conformal Lower Bound for the Smallest Eigenvalue of the Dirac Operator and Killing Spinors
}

\author{
Oussama Hijazi \\ Max-Planck-Institut für Mathematik, Gottfried-Claren-Strasse 26, D-5300 Bonn 3, \\ Federal Republic of Germany
}

\begin{abstract}
On a Riemannian spin manifold, we give a lower bound for the square of the eigenvalues of the Dirac operator by the smallest eigenvalue of the conformal Laplacian (the Yamabe operator). We prove, in the limiting case, that the eigenspinor field is a killing spinor, i.e., parallel with respect to a natural connection. In particular, if the scalar curvature is positive, the eigenspinor field is annihilated by harmonic forms and the metric is Einstein.
\end{abstract}

\section{Introduction}

In 1963 Lichnerowicz [12] proved that on a Riemannian spin manifold the square of the Dirac operator $\mathscr{D}$ is given by

$$
\mathscr{D}^{2}=\Delta+\frac{s}{4}
$$

where $\Delta$ is the positive spinor Laplacian and $s$ the scalar curvature. This formula implies

Theorem [12]. On a compact Riemannian spin manifold $(M, g)$ of positive scalar curvature,

(i) there is no non-zero harmonic spinor, and

(ii) any eigenvalue $\lambda$ of the Dirac operator satisfies

$$
\lambda^{2}>\frac{1}{4} \inf _{M} s .
$$

Part (i) together with the Atiyah-Singer index theorem applied to the Dirac operator for $4 k$-dimensional manifolds, gives a topological obstruction - namely the vanishing of the $\hat{A}$-genus of Hirzebruch - for the existence of positive scalar curvature metrics on a compact spin manifold.

Hitchin [7] extended Lichnerowicz' result of the vanishing of the $\mathrm{KO}$ characteristic numbers defined by Milnor [13]. By introducing the notion of enlargeable manifolds and combining it with the spin condition, Gromov and 
Lawson [4-6] obtained beautiful results for the non-existence (and existence) of positive scalar curvature metrics on certain compact (and non-compact) manifolds. It should be mentioned that in an earlier paper, Schoen and Yau [14] used the technique of regular minimal hypersurfaces to prove that, in low dimensions, certain manifolds do not support positive scalar curvature metrics.

\section{Main Results}

Theorem A. Let $(M, g)$ be a compact Riemannian spin manifold of dimension $n \geqq 3$. Any eigenvalue $\lambda$ of the Dirac operator $\mathscr{D}$ satisfies

$$
\lambda^{2} \geqq \frac{n}{4(n-1)} \mu_{1}
$$

where $\mu_{1}$ is the first eigenvalue of the Yamabe operator $L$,

$$
L \equiv 4 \frac{n-1}{n-2} \Delta+s
$$

where $\Delta$ is the positive Laplacian acting on functions and $s$ the scalar curvature of $(M, g)$.

Theorem B. Let $(M, g)$ be a compact Riemannian spin manifold of dimension $n \geqq 3$. If there exists an eigenspinor field $\Psi_{1}$ of the Dirac operator $\mathscr{D}$ for the eigenvalue $\lambda_{1}$, with $\lambda_{1}^{2}=\frac{n}{4(n-1)} \mu_{1}$, then either i) or ii) holds:

i) $\mu_{1}=0$, then there exists a function $u_{1}$ satisfying

$$
4 \frac{n-1}{n-2} \Delta\left(e^{-\frac{n-2}{2} u_{1}}\right)+s e^{-\frac{n-2}{2} u_{1}}=0
$$

such that, with respect to the metric $\bar{g}=e^{2 u_{1}} g$ and its corresponding connection $\bar{\nabla}$, there exists a parallel spinor field $\bar{\Phi}_{1}$. In particular, the manifold $(M, \bar{g})$ is Ricci-flat.

ii) $\mu_{1}>0$, then $\nabla^{\lambda_{1}} \Psi_{1} \equiv 0$, where for a real function $f$, a vector field $X$ and $a$ spinor field $\Psi$, the modified connection $\nabla^{f}$ is defined by

$$
\nabla_{X}^{f} \Psi \equiv \nabla_{X} \Psi+\frac{f}{n} X \cdot \Psi
$$

(Here, "." denotes Clifford multiplication.) In particular, the manifold $(M, g)$ is Einstein.

It should be pointed out that Friedrich [2] proved that

$$
\lambda^{2} \geqq \frac{n}{4(n-1)} \inf _{M} s,
$$

and that equality gives an Einstein metric. One can easily see that $\mu_{1} \geqq \inf _{M} s$ [see Remark 5.8(b) below]. It is a well known fact [9] that the sign of $\mu_{1}$ is a conformal 
invariant. In Inequality (2.1), instead of $\mu_{1}$, one can put the Yamabe number which is another conformal invariant. Let $\bar{g}$ be any metric in the conformal class of $g$ and denote by $\bar{\nabla}$ its Levi-Civita connection. The basic idea in the proof of Theorem A is to write the Lichnerowicz formula for a family of modified connections $\bar{\nabla}^{f}$, and then to apply it to a spinor field lying in the kernel of the corresponding modified Dirac operator.

Now, define a map

$$
\mathscr{R i c}{ }^{f}: T M \otimes \Sigma M \rightarrow \Sigma M
$$

by

$$
\mathscr{R} i c^{f}(X \otimes \Psi) \equiv \sum_{i=1}^{n} e_{i} \cdot \mathscr{R}_{X, e_{i}}^{f} \Psi,
$$

where $\left(e_{1}, \ldots, e_{n}\right)$ is an orthonormal basis of the tangent bundle $T M$ and $\mathscr{R}^{f}$ is the curvature form of a family of modified connections $\nabla^{f}$ and acting on the spinor bundle $\Sigma M$.

Proposition C. Let $(M, g)$ be a Riemannian spin manifold of dimension $n \geqq 3$. The manifold $(M, g)$ is Einstein with positive scalar curvature s if and only if for some non-trivial real function $f$ and for any tangent vector $X$, the endomorphism $\mathscr{R}_{i}{ }_{X}^{f}$ is non-invertible. In this case $f^{2}=\frac{n}{4(n-1)}$ s.

In Sect. 3 we prove Proposition $C$ and deduce that the existence of a non-trivial $\nabla^{f}$-parallel spinor field (what is called a Killing spinor in physics) implies that the metric is Einstein and $f^{2}=\frac{n}{4(n-1)} s$.

In Sect. 4 we give the relation between the Dirac operators associated with two conformally related metrics [7], and in Sect. 5 we give the proof of Theorem A.

In Sect. 6 we study the limiting case of Inequality (2.1). We start by proving Theorem B, according to which Killing spinors are characterized as the eigenspinor fields associated with the limiting eigenvalues of the Dirac operator. Then we give some geometric consequences. In particular, we prove that in the limiting case, eigenspinor fields are killed by harmonic forms and even-dimensional manifolds are Einstein non-Kähler. Finally, we give some rigidity results in dimensions 4 and 6 .

\section{Spinor Characterization of Riemannian Positive Einstein Spin Manifolds}

For an introduction to Clifford algebras and spin geometry we refer to $[1,11]$. We start this section by the following computational lemma.

Lemma 3.1. Let $\alpha$ be an exterior $k$-form and $\Psi$ any non-trivial complex spinor. Then, the inner product

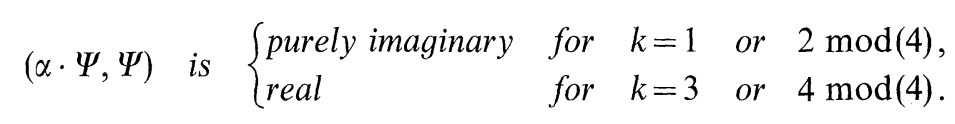


Proof. Take $\alpha$ to be of the form $\alpha=e_{1} \cdot e_{2} \ldots e_{k}$, where $\left(e_{1}, e_{2}, \ldots, e_{n}\right)$ is an orthonormal basis. Then,

$$
\begin{aligned}
\left(e_{1} \cdot e_{2} \ldots e_{k} \cdot \Psi, \Psi\right) & =(-1)^{k}\left(\Psi, e_{k} \ldots e_{2} \cdot e_{1} \cdot \Psi\right) \\
& =(-1)^{k}(-1)^{\frac{k(k-1)}{2}}\left(\Psi, e_{1} \cdot e_{2} \ldots e_{k} \cdot \Psi\right) \\
& =(-1)^{\frac{k(k+1)}{2}}\left(\overline{e_{1} \cdot e_{2} \ldots e_{k} \cdot \Psi, \Psi}\right)
\end{aligned}
$$

We consider a real function $f$ on a Riemannian spin manifold $(M, g)$ of dimension $n \geqq 3$. We then define a family of modified connections $\nabla^{f}$ by setting

$$
\nabla_{X}^{f} \Psi \equiv \nabla_{X} \Psi+\frac{f}{n} X \cdot \Psi
$$

for any vector field $X$ and any spinor field $\Psi$, where $\nabla$ denotes the Levi-Civita spinor connection and "." is the Clifford multiplication.

A direct computation using the definition of $\nabla^{f}$ and the compatibility of the covariant derivative with the Clifford multiplication, gives for any tangent vector $X$ and any spinor $\Psi$ the relation

$$
\mathscr{R} i^{f}(X \otimes \Psi)=-\frac{1}{2} \operatorname{Ric}(X) \cdot \Psi+\frac{2(n-1)}{n^{2}} f^{2} X \cdot \Psi-\frac{1}{n} \operatorname{grad} f \cdot X \cdot \Psi-X(f) \Psi,
$$

where $\mathscr{R} i c^{f}$ is the zero-order operator defined by (2.3), Ric is the Ricci tensor viewed as a map of the tangent bundle and $X(f)$ is the Lie derivative of the function $f$ in the direction of $X$.

3.4. Proof of Proposition $C$. If for all vectors $X$ one can find a non-zero spinor $\Psi$ such that

$$
\mathscr{R i c}^{f}(X \otimes \Psi)=0,
$$

then by taking $X=\operatorname{grad} f$ in Eq. (3.3) we get

$$
\left(\frac{2(n-1)}{n^{2}} f^{2} \operatorname{grad} f-\frac{1}{2} \operatorname{Ric}(\operatorname{grad} f)\right) \cdot \Psi=\frac{n-1}{n}|\operatorname{grad} f|^{2} \Psi .
$$

Now, taking the inner product of this equation with $\Psi$ and using Lemma 3.1 we get

$$
|\operatorname{grad} f|^{2}=0 \text {. }
$$

The function $f$ is then constant and Eq. (3.3) - applied, for each tangent vector $X$, to the appropriate spinor $\Psi$-implies that

$$
\operatorname{Ric}(X)=4 \frac{n-1}{n^{2}} f^{2} X
$$

hence $g$ is an Einstein metric and $f^{2}=\frac{n}{4(n-1)} s$. 
Corollary 3.6. If $\Psi$ is a non-trivial spinor field with $\nabla^{f} \Psi \equiv 0$, then $f$ is constant and the manifold is Einstein with $f^{2}=\frac{n}{4(n-1)}$ s. In particular, if $\nabla \Psi \equiv 0$, then the manifold is Ricci-flat.

Remark 3.7. On a compact 4-dimensional manifold the existence of a non-trivial parallel spinor field implies that [8] the manifold is either the flat torus or a $K 3$ surface.

\section{Dirac Operators of Conformally Related Metrics}

In this section we consider a conformal change of the Riemannian metric. Using the relation between the Levi-Civita connections on the tangent bundle corresponding to two conformally related metrics, we relate the two canonical spinor connections acting on two isometric spinor bundles. This enables us to compare the Dirac operators.

\subsection{Isometry of the Spinor Bundles Associated with Two Conformally Related Metrics}

Let $(M, g)$ be a Riemannian spin manifold of dimension $n \geqq 3$. Consider a metric $\bar{g}$ pointwise conformal to $g$, i.e., $\bar{g}=e^{2 u} g$ for some real function $u$ on $M$. We denote by $\mathrm{SO}_{g} M$ (respectively $\mathrm{SO}_{\bar{g}} M$ ) the bundle of $g$-orthonormal (respectively $\bar{g}$-orthonormal) frames. Locally, to a section $\varepsilon=\left(X_{1}, \ldots, X_{n}\right)$ of $\mathrm{SO}_{g} M$ corresponds a section $\bar{\varepsilon}=\left(e^{-u} X_{1}, \ldots, e^{-u} X_{n}\right)$ of $\mathrm{SO}_{\bar{g}} M$. This isometry will be denoted by $G_{u}$.

Let $\gamma$ be a spin structure on $(M, g)$. We denote by $\operatorname{Spin}_{\gamma} M$ the $\operatorname{Spin}_{n}$-principal bundle associated with it which is fiberwise a non-trivial double covering of $\mathrm{SO}_{g} M$. Thanks to the isomorphism $G_{u}$ one can define a spin structure $\bar{\gamma}$ on $(M, \bar{g})$ in such a way that the diagram

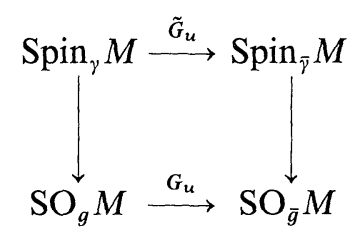

commutes.

To an irreducible representation $\Sigma_{n}$ of $\operatorname{Spin}_{n}$ one associates two isometric spinor bundles $\Sigma M$ and $\Sigma \bar{M}$ by taking the vector bundles associated with the isomorphic $\operatorname{Spin}_{n}$-principal bundles, $\operatorname{Spin}_{\gamma} M$ and $\operatorname{Spin}_{\bar{\gamma}} M$.

For any section $\Psi$ of $\Sigma M$, we write $\bar{\Psi} \equiv G_{u} \Psi$. Clifford multiplication in $\Sigma \bar{M}$ is given, for a vector field $X$ and a spinor field $\Psi$, by

$$
\bar{X}-\bar{\Psi}=\overline{X \cdot \Psi} \text {. }
$$




\subsection{Comparison of the Spinor Connections}

Proposition 4.2.1. The Levi-Civita spinor connections $\nabla$ and $\bar{\nabla}$ acting respectively on the sections of $\Sigma M$ and $\Sigma \bar{M}$, are related, for any vector field $X$ and any spinor field $\Psi, b y$

$$
\bar{\nabla}_{X} \bar{\Psi}=\overline{\nabla_{X} \Psi}-\frac{1}{2} \overline{X \cdot \operatorname{grad} u \cdot \Psi}-\frac{1}{2} X(u) \bar{\Psi},
$$

where $X(u)$ is the Lie derivative of the function $u$ in the direction of $X$.

Proof. Let $\varepsilon=\left(X_{1}, \ldots, X_{n}\right)$ be a local $g$-orthonormal tangent frame field and denote by $\omega$ and $\bar{\omega}$, the connection forms corresponding to $g$ and $\bar{g}$. For all indices $i, j$ and $k$ in $\{1, \ldots, n\}$, we have

$$
\bar{\omega}_{i j}\left(X_{k}\right)=\omega_{i j}\left(X_{k}\right)+X_{i}(u) \delta_{k j}-X_{j}(u) \delta_{k i} .
$$

Locally, the choice of $\tilde{\varepsilon}$ in $\operatorname{Spin}_{\gamma} M$ of image $\varepsilon$ in $\mathrm{SO}_{g} M$ determines a local frame field $\left(\sigma_{1}, \ldots, \sigma_{N}\right)$ of $\Sigma M$, where $N=2^{[n / 2]}$, such that for all indices $i, j$ and $k$ in $\{1, \ldots, n\}$ and $A$ in $\{1, \ldots, N\}$, the spinor covariant derivative of $\sigma_{A}$ is given [11] by

$$
\nabla_{X_{k}} \sigma_{A}=\frac{1}{2} \sum_{i<j} \omega_{i j}\left(X_{k}\right) X_{i} \cdot X_{j} \cdot \sigma_{A} \text {. }
$$

With respect to $\bar{g}$, we get

$$
\begin{aligned}
\bar{\nabla}_{X_{k}} \bar{\sigma}_{A} & =\frac{1}{2} \sum_{i<j}\left(\omega_{i j}\left(X_{k}\right)+X_{i}(u) \delta_{k j}-X_{j}(u) \delta_{i k}\right) \overline{X_{i} \cdot X_{j} \cdot \sigma_{A}} \\
& =\overline{\bar{\nabla}_{X_{k}} \sigma_{A}}+\frac{1}{2} \sum_{i<k} X_{i}(u) \overline{X_{i} \cdot X_{k} \cdot \sigma_{A}}-\frac{1}{2} \sum_{k<j} X_{j}(u) \overline{X_{k} \cdot X_{j} \cdot \sigma_{A}}
\end{aligned}
$$

hence,

$$
\bar{\nabla}_{X_{k}} \bar{\sigma}_{A}=\overline{\bar{\nabla}_{X_{k}} \sigma_{A}}-\frac{1}{2} \overline{X_{k} \cdot \operatorname{grad} u \cdot \sigma_{A}}-\frac{1}{2} X_{k}(u) \overline{\sigma_{A}} .
$$

\subsection{Comparison of the Dirac Operators}

Proposition 4.3.1. Let $(M, g)$ be an $n$-dimensional Riemannian spin manifold. The Dirac operators $\mathscr{D}$ and $\mathscr{D}$, respectively associated with the metrics $g$ and $\bar{g}=e^{2 u} g$ and acting respectively on the sections of the spinor bundles $\Sigma M$ and $\Sigma \bar{M}$, satisfy

$$
\overline{\mathscr{D}}\left(e^{-\frac{n-1}{2} u} \bar{\Psi}\right)=e^{-\frac{n+1}{2} u} \overline{\mathscr{D} \Psi}
$$

for any spinor field $\Psi$ of $\Sigma M$.

Proof. Take $\varepsilon=\left(X_{1}, \ldots, X_{n}\right)$ to be a local section of $\mathrm{SO}_{g} M$ and $\bar{\varepsilon}=\left(e^{-u} X_{1}, \ldots, e^{-u} X_{n}\right)$ its image in $\mathrm{SO}_{\bar{g}} M$ under $G_{u}$. Locally, the Dirac operator $\overline{\mathscr{D}}$ is given by

$$
\overline{\mathscr{D}} \bar{\Psi}=\sum_{k=1}^{n} \bar{X}_{k}-\bar{\nabla}_{X_{k}} \bar{\Psi} .
$$

Using Eq. (4.2.2) we get

$$
\overline{\mathscr{D}} \bar{\Psi}=e^{-u}\left(\overline{\mathscr{D} \Psi}+\frac{n-1}{2} \overline{\operatorname{grad} u \cdot \Psi}\right) .
$$


On the other hand, for any real function $f$, one has

$$
\overline{\mathscr{D}}(f \bar{\Psi})=f \overline{\mathscr{D}} \bar{\Psi}+e^{-u} \overline{\operatorname{grad} f \cdot \Psi},
$$

which, with Eq. (4.3.3) and for $f=e^{-\frac{n-1}{2} u}$, gives Eq. (4.3.2).

Remark. It is a classical result [7] that the dimension of the space of harmonic spinors is a conformal invariant. Indeed, by Eq. (4.3.2), if $\mathscr{D} \Psi=0$, then $\overline{\mathscr{D}} \bar{\Phi}=0$ where $\bar{\Phi}=e^{-\frac{n-1}{2} u} \bar{\Psi}$, and conversely.

\section{Proof of the Basic Inequality (Theorem A)}

It is clear that Inequality (2.1) is only of interest for the eigenvalues of $\mathscr{D}$ with the smallest absolute value, and on a Riemannian manifold for which $\mu_{1}$ is nonnegative. This condition turns out to be equivalent to the existence of a conformally equivalent metric with non-negative scalar curvature [10].

We first prove that for any positive function $h$,

$$
\lambda^{2} \geqq \frac{n}{4(n-1)} \inf _{M}\left(4 \frac{n-1}{n-2} h^{-1} \Delta h+s\right),
$$

then we take $h=h_{1}$ to be an eigenfunction for the first eigenvalue of the Yamabe operator $\mu_{1}$ (it is known that the eigenspace of $\mu_{1}$ is 1 -dimensional and contains functions which do not change sign) so we get

$$
\lambda^{2} \geqq \frac{n}{4(n-1)} \mu_{1}
$$

For $n \geqq 3$, it is convenient to write the conformal change

$$
e^{2 u}=h^{\frac{4}{n-2}} \text { for a positive function } h \text {. }
$$

It is well known that the scalar curvature $\bar{s}\left(\equiv s_{h}\right)$ of the metric $\bar{g}=e^{2 u} g\left(\equiv \frac{4}{h^{n-2}} g\right)$ is related to the scalar curvature $s$ of the metric $g$ by

or equivalently

$$
s_{h} h^{\frac{n+2}{n-2}}=4 \frac{n-1}{n-2} \Delta h+s h
$$

$$
s_{h} \frac{4}{h^{n-2}}=4 \frac{n-1}{n-2} h^{-1} \Delta h+s .
$$

Now, let us consider the family of connections $\nabla^{f}$ defined by (3.2). The associated family of Dirac operators $\mathscr{D}^{f}$ acting on a spinor field $\Psi$, is given by

$$
\mathscr{D}^{f} \Psi=\sum_{i} X_{i} \cdot \nabla_{X_{i}}^{f} \Psi=(\mathscr{D}-f) \Psi
$$


Taking the square of this operator, one gets

$$
(\mathscr{D}-f)^{2} \Psi=\mathscr{D}^{2} \Psi-2 f \mathscr{D} \Psi-\operatorname{grad} f \cdot \Psi+f^{2} \Psi .
$$

By Lichnerowicz' formula (1.1) and a straightforward computation using the definitions of $\nabla^{f}$ and the corresponding Laplacian $\Delta^{f}$, we get

$$
(\mathscr{D}-f)^{2} \Psi=\Delta^{f} \Psi+\left(\frac{s}{4}+\frac{n-1}{n} f^{2}\right) \Psi-\frac{n-1}{n}(2 f \mathscr{D} \Psi+\operatorname{grad} f \cdot \Psi) .
$$

We now consider a conformal change of metric, $\bar{g}=e^{2 u} \mathrm{~g}$, and we take $\Psi$ such that $\mathscr{D} \Psi=\lambda \Psi$. By Eq. (4.3.2) we get

$$
\overline{\mathscr{D}} \bar{\Phi}=\lambda e^{-u} \bar{\Phi},
$$

where

$$
\Phi=e^{-\frac{n-1}{2} u} \Psi
$$

Equation (5.4) written with respect to the metric $\bar{g}$ and applied to $\bar{\Phi}$ gives, after taking the $\bar{g}$-global inner product on $(M, \bar{g})$, the equation

$$
\begin{aligned}
\int_{M} \bar{g}\left((\overline{\mathscr{D}}-f)^{2} \bar{\Phi}, \bar{\Phi}\right) v_{\bar{g}}= & \int_{M} \bar{g}\left(\overline{V^{f}} \bar{\Phi}, \bar{\nabla}^{f} \bar{\Phi}\right) v_{\bar{g}}+\int_{M}\left(\frac{\bar{s}}{4}+\frac{n-1}{n} f^{2}\right) \bar{g}(\bar{\Phi}, \bar{\Phi}) v_{\bar{g}} \\
& -\frac{n-1}{n} \int_{M} \bar{g}\left(2 f \overline{\mathscr{D}} \bar{\Phi}+e^{-u} \overline{\operatorname{grad} f \cdot \Phi}, \bar{\Phi}\right) v_{\bar{g}} .
\end{aligned}
$$

We choose $f=\lambda e^{-u}$, so that $\overline{\mathscr{D}} \bar{\Phi}=f \bar{\Phi}$. The left-hand side of Eq. (5.6) is then zero and by Lemma 3.1 the function $\bar{g}(\overline{\operatorname{grad} f \cdot \Phi}, \bar{\Phi})$ is purely imaginary, hence

$$
0=\int_{M} \bar{g}\left(\bar{\nabla}^{f} \bar{\Phi}, \bar{\nabla}^{f} \bar{\Phi}\right) v_{\bar{g}}+\int_{M}\left(\frac{\bar{s}}{4}-\frac{n-1}{n} f^{2}\right) \bar{g}(\bar{\Phi}, \bar{\Phi}) v_{\bar{g}}, \quad \text { with } \quad f=\lambda e^{-u}
$$

Since the first term in (5.7) is non-negative, the second term must be non-positive. A necessary condition then is that, for any function $u$

$$
\lambda^{2} \geqq \frac{n}{4(n-1)} \inf _{M}\left(\bar{s} e^{2 u}\right),
$$

which combines with (5.2) and (5.3) to give the desired result.

Remark 5.8. (a) In fact, one can prove that indeed,

$$
\mu_{1}=\sup _{h>0} \inf _{M}\left(4 \frac{n-1}{n-2} h^{-1} \Delta h+s\right) .
$$

To see this, take $h_{1}>0$ to be an eigenfunction for $\mu_{1}$ of the Yamabe operator (2.2) and consider for all functions $h>0$, the number

$$
\inf _{M}\left\{\left(4 \frac{n-1}{n-2} h^{-1} \Delta h+s\right)-\left(4 \frac{n-1}{n-2} h_{1}^{-1} \Delta h_{1}+s\right)\right\} .
$$


To prove that this number is non-positive, it is sufficient to prove that the integral of $\left(h^{-1} \Delta h-h_{1}^{-1} \Delta h_{1}\right)$ with respect to some positive measure $v$ is non-positive. We take $v=h_{1}^{2} v_{g}$, so that

$$
\int_{M}\left(h^{-1} \Delta h-h_{1}^{-1} \Delta h_{1}\right) h_{1}^{2} v_{g}=-\int_{M} h^{2}\left|d\left(h_{1} h^{-1}\right)\right|^{2} v_{g} \leqq 0 .
$$

(b) Taking $h$ to be a constant in (5.9), one gets $\inf _{M} s<\mu_{1}$, unless $s$ is a constant in which case one has equality.

\section{The Limiting Case of the Inequality}

In this section we will be concerned with the properties of compact Riemannian spin manifolds of dimension $n \geqq 3$, which admit $\Psi_{1}$ such that $\mathscr{D} \Psi_{1}=\lambda_{1} \Psi_{1}$, with $\lambda_{1}^{2}=\frac{n}{4(n-1)} \mu_{1}$.

\subsection{Proof of Theorem $B$}

Consider Eq. (5.7) for the metric $\bar{g}=e^{2 u_{1}} g$, where $u_{1}$ is the function corresponding to a positive eigenfunction $h_{1}$ associated with the first eigenvalue $\mu_{1}$ for the Yamabe operator (2.2). Since $\lambda_{1}^{2}=\frac{n}{4(n-1)} \mu_{1}$, the second term in (5.7) vanishes,
hence

$$
\bar{\nabla}^{f_{1}} \bar{\Phi}_{1} \equiv 0 \quad \text { with } \quad f_{1} \equiv \lambda_{1} e^{-u_{1}} .
$$

Now two cases can occur:

(i) If $\mu_{1}=0\left(\lambda_{1}=0\right)$, then $\bar{\nabla} \bar{\Phi}_{1} \equiv 0$. By Corollary 3.6, the manifold $\left(M, \bar{g}=e^{2 u_{1}} g\right)$ is Ricci-flat.

(ii) If $\mu_{1}>0\left(\lambda_{1} \neq 0\right)$, again by Corollary 3.6, Eq. (6.1) implies that $u_{1}$ must be constant. Using Eq. (4.2.2) we get

$$
\nabla^{\lambda_{1}} \Psi_{1} \equiv 0
$$

hence by Corollary 3.6, the manifold $(M, g)$ is Einstein.

Proposition 6.2. Let $(M, g)$ be a compact Riemannian spin manifold of dimension $n \geqq 3$. Let $\Psi_{1}$ be an eigenspinor field associated with $\lambda_{1}$ such that $\lambda_{1}^{2}=\frac{n}{4(n-1)} \mu_{1}$,
then two cases can occur:

(i) $\mu_{1}=0$, then with respect to the metric $\bar{g}=e^{2 u_{1}}$ g, the space of harmonic spinors is stable under Clifford multiplication by harmonic forms.

(ii) $\mu_{1}>0$, then any harmonic form kills the eigenspinor $\Psi_{1}$.

Proof. Let $\beta$ be a homogenous $k$-form and $\Psi$ a spinor field, then

hence

$$
\mathscr{D}(\beta \cdot \Psi)=\sum_{i} X_{i} \cdot \nabla_{X_{i}}(\beta \cdot \Psi)=\sum_{i} X_{i} \cdot \nabla_{X_{i}} \beta \cdot \Psi+\sum_{i} X_{i} \cdot \beta \cdot \nabla_{X_{i}} \Psi,
$$

$$
\mathscr{D}(\beta \cdot \Psi)=((d+\delta) \beta) \cdot \Psi+\sum_{i} X_{i} \cdot \beta \cdot \nabla_{X_{i}} \Psi,
$$

where $d$ is the exterior differential and $\delta$ its adjoint. 
Case (i) $\mu_{1}=0$. We consider Eq. (6.2.1) for the metric $\bar{g}=e^{2 u_{1}} g$ and apply it to the spinor field $\bar{\Phi}_{1}=e^{-\frac{n-1}{2} u_{1}} \bar{\Psi}_{1}$ and use Theorem Bi) in order to conclude (i).

Case (ii) $\mu_{1}>0$. Then by Theorem Bii), for any vector field $X$, one has

$$
\nabla_{X} \Psi_{1}=-\frac{\lambda_{1}}{n} X \cdot \Psi_{1}
$$

By a direct check one sees that for homogenous $k$-form $\beta$,

$$
\sum_{i} X_{i} \cdot \beta \cdot X_{i}=(-1)^{k-1}(n-2 k) \beta
$$

hence Eq. (6.2.1) applied to $\Psi_{1}$ gives

$$
\mathscr{D}\left(\beta \cdot \Psi_{1}\right)=((d+\delta) \beta) \cdot \Psi_{1}+(-1)^{k} \lambda_{1}\left(1-\frac{2 k}{n}\right) \beta \cdot \Psi_{1} .
$$

If $\beta$ is harmonic, then the spinor field $\beta \cdot \Psi_{1}$ if non-zero would be an eigenspinor field for the eigenvalue $(-1)^{k} \lambda_{1}\left(1-\frac{2 k}{n}\right)$ which, for $\lambda_{1} \neq 0$, has absolute value less
than $\lambda_{1}$. Thus $\beta \cdot \Psi_{1}=0$. $\square$

Remark. If $\beta$ is a harmonic 1 -form, then $\beta \cdot \Psi_{1}=0$ implies that $\beta=0$, i.e., the first Betti number is zero (this is a known result since the manifold is Einstein with positive scalar curvature).

Proposition 6.3. Let $f$ be a non-trivial real function on an even-dimensional spin manifold. If there exists a non-trivial $\nabla^{f}$-parallel spinor field $\Psi$, then the manifold is Einstein non-Kähler.

Proof. By Corollary 3.6, $\nabla^{f} \Psi \equiv 0$ implies that the manifold is Einstein and $f^{2}=\frac{n}{4(n-1)} s$, hence $\Psi$ is an eigenspinor field of the Dirac operator for the eigenvalue $\lambda_{1}$, with $\lambda_{1}^{2}=\frac{n}{4(n-1)} \mu_{1}$.

Suppose that the manifold is Kähler and denote by $\Omega$ its Kähler form. Since $\Omega$ is harmonic, by Proposition 6.2(ii), we get $\Omega \cdot \Psi=0$. It is a direct check that

$$
\Omega \cdot \Omega \cdot \Psi=(\Omega \wedge \Omega) \cdot \Psi-\frac{n}{2} \Psi .
$$

Since $\Omega \cdot \Omega \cdot \Psi=(\Omega \wedge \Omega) \cdot \Psi=0$ and $\Psi \neq 0$, there is a contradiction.

On an even-dimensional spin manifold the spectrum of the Dirac operator is symmetric about 0 . In fact, in these dimensions, any spinor field $\Psi$ splits as the sum of a positive spinor $\Psi^{+}$and a negative spinor $\Psi^{-}$. So, if $\mathscr{D} \Psi=\lambda \Psi$, then $\widetilde{\Psi} \equiv \Psi^{+}-\Psi^{-}$is such that $\mathscr{D} \widetilde{\Psi}=-\lambda \widetilde{\Psi}$.

Theorem 6.4. Let $(M, g)$ be a compact Riemannian spin manifold of dimension 4 . If there exists an eigenspinor field $\Psi_{1}$ for $\lambda_{1}$ with $\lambda_{1}^{2}=\frac{\mu_{1}}{3}>0$, then the manifold $(M, g)$ is isometric to $\left(S^{4}\right.$, can $)$.

This theorem has been proved by Friedrich [3] using a different method. 
Proof. By Theorem B(ii) we have that the Ricci tensor field is given by Ric $=\frac{S}{4} \mathrm{Id}$. We write $\Psi_{1}=\Psi_{1}^{+}+\Psi_{1}^{-}$and consider $\tilde{\Psi}_{1}=\Psi_{1}^{+}-\Psi_{1}^{-}$. The function $f \equiv\left(\Psi_{1}, \widetilde{\Psi}_{1}\right)$ $=\left|\Psi_{1}^{+}\right|^{2}-\left|\Psi_{1}^{-}\right|^{2}$ satisfies

$$
\Delta f=\frac{s}{3} f
$$

(the straightforward proof relies on the fact that $\nabla^{\lambda_{1}} \Psi_{1}=\nabla^{-\lambda_{1}} \widetilde{\Psi}_{1} \equiv 0$ ).

Now, the Obata-Lichnerowicz theorem says that if on an $n$-dimensional compact Riemannian manifold there exists a non-zero function $f$ and a positive constant $c$ such that,

$$
\text { Ric } \geqq c \text { Id } \text { and } \quad \Delta f=\frac{n}{n-1} c f,
$$

then the manifold is isometric to $\left(S^{n}\right.$, can).

In order to apply this theorem we need only to prove that the function $f \equiv\left(\Psi_{1}, \widetilde{\Psi}_{1}\right)$ does not vanish identically. One can see this as follows: using $\nabla^{\lambda_{1}} \Psi_{1}$ $=\nabla^{-\lambda_{1}} \widetilde{\Psi}_{1} \equiv 0$, for any vector field $X$, one has

$$
X(f)=\lambda_{1} \operatorname{Re}\left(X \cdot \Psi_{1}^{+}, \Psi_{1}^{-}\right)
$$

Let us consider now the $\mathbb{R}$-linear injective map

$$
T M \rightarrow \Sigma^{-} M, \quad X \rightarrow X \cdot \Psi_{1}^{+},
$$

where $\Sigma^{-} M$ is the space of negative spinors. In a 4-dimensional spin manifold, one has

$$
\operatorname{dim}_{\mathbb{R}} \Sigma^{-} M=\operatorname{dim}_{\mathbb{R}} T M=4,
$$

hence $T M$ is isomorphic to $\Sigma^{-} M$ and there exists $X \neq 0$ such that

$$
\left(X \cdot \Psi_{1}^{+}, \Psi_{1}^{-}\right) \neq 0
$$

which implies that $f \neq 0$.

Proposition 6.5. Let $(M, g)$ be a 6-dimensional compact Riemannian spin manifold. If the eigenspinor space of $\lambda_{1}$, with $\lambda_{1}^{2}=\frac{3}{10} \mu_{1}>0$, is 2-dimensional, then $(M, g)$ is isometric to $\left(S^{6}\right.$, can $)$.

Proof. As in Theorem 6.4 it is sufficient to prove that the function $f \equiv\left|\Psi_{1}^{+}\right|^{2}-\left|\Psi_{1}^{-}\right|^{2}$ is not identically zero, where $\Psi_{1}$ is such that $\mathscr{D} \Psi_{1}=\lambda_{1} \Psi_{1}$, with $\lambda_{1}^{2}=\frac{3}{10} \mu_{1}>0$. For a 6-dimensional manifold, we have $\operatorname{dim}_{\mathbb{C}} \Sigma^{-} M=4$. If for all vectors $X$, the spinor $X \cdot \Psi_{1}^{+}$lies in a 2-dimensional complex space there will be a contradiction with the fact that the map (6.4.1) is injective, so $f \neq 0$.

Acknowledgements. The author wishes to thank Prof. J. P. Bourguignon and P. Gauduchon for their continuing help and encouragement, without which this paper would not exist.

This work is an extension of the author's "thèse de $3^{\circ}$ cycle," prepared in the "Centre de Mathématiques de l'Ecole Polytechnique." 


\section{References}

1. Atiyah, M.F., Bott, R., Shapiro, A.: Clifford modules. Topology 3 [Suppl. 1], 3-38 (1964)

2. Friedrich, T.: Der erste Eigenwert des Dirac-Operators einer kompakten, Riemannschen Mannigfaltigkeit nicht negativer Skalarkrümmung. Math. Nachr. 97, 117-146 (1980)

3. Friedrich, T.: A remark on the first eigenvalue of the Dirac operator on 4-dimensional manifolds. Math. Nachr. 102, 53-56 (1981)

4. Gromov, M., Lawson, H.B.: Spin and scalar curvature in the presence of a fundamental group. I. Ann. Math. 111, 209-230 (1980)

5. Gromov, M., Lawson, H.B.: The classification of simply connected manifolds of positive scalar curvature. Ann. Math. 111, 423-434 (1980)

6. Gromov, M., Lawson, H.B.: Positive scalar curvature and the Dirac operator on complete Riemannian manifolds. I.H.E.S. 58, 295-408 (1983)

7. Hitchin, N.: Harmonic spinors. Adv. Math. 14, 1-55 (1974)

8. Hitchin, N.: Compact four-dimensional Einstein manifolds. J. Differ. Geom. 9, 435-441 (1974)

9. Kazdan, J.L., Warner, F.W.: Scalar curvature and conformal deformations of Riemannian structures. J. Differ. Geom. 10, 113-134 (1975)

10. Kazdan, J.L., Warner, F.W.: Prescribing curvatures, Proceedings of Symposia in Pure Mathematics, 27 (1975)

11. Lawson, H.B., Michelsohn, M.L.: Spin geometry. Universidade Federal do Ceara (1983)

12. Lichnerowicz, A.: Spineurs harmoniques. C.R. Acad. Sci. Paris Ser. A-B, 257 (1963)

13. Milnor, J.: Remarks concerning spin manifolds. Differential and combinatorial topology: A symposium in Honor of Marston Morse, pp. 55-62. Princeton, NJ: Princeton University Press 1965

14. Schoen, R., Yau, S.-T.: On the structure of manifolds with positive scalar curvature. Manuscr. Math. 28, 159-183 (1979)

Communicated by A. Jaffe

Received August 28, 1985 